\section{Effect of "backward" masker fringe on the detectability of pulsed diotic and dichotic tonal signals}

\author{
CONSTANTINE TRAHIOTIS \\ University of Illinois, Champaign, Illinois 61820 \\ and \\ TERRENCE R. DOLAN and THEODORE H. MILLER \\ Parmly Hearing Institute and Loyola University, Chicago, Illinois 60626
}

The effects of backward masker "fringe" on performance in homophasic and antiphasic masking conditions were investigated. The results of the study indicate that (1) the presence of a backward masker fringe has only a small effect on performance in homophasic masking conditions; (2) under antiphasic masking conditions, the presence of a backward masker fringe improves performance; (3) similar to the results of studies investigating the effects of forward masker fringe, the magnitude of the improvement in performance increases as the duration of the fringe increases; and (4) the magnitude of the improvement caused by the presence of backward masker fringe is considerably smaller than the magnitude of the improvement caused by the forward masker fringe. It appears, then, that the presence of backward masker fringe may provide a baseline or reference phase similar to that which the forward fringe is presumed to provide. The presence of this reference phase after the offset of the signal apparently makes the phase shift associated with the signal-plus-noise waveform more discernible, thus leading to higher detectability. However, for reasons not yet clear, the presence of a baseline or reference phase after the offset of the signal (backward fringe conditions) does not make the signal-plus-noise phase shift as discernible as do either the presence of that same reference phase before the onset of the signal (forward fringe condition) or the presence of that same reference phase both before signal onset and after signal of fset (continuous masker condition).

Most of the investigations of binaural masking have employed continuous noise as the masker and a gated tone as the signal (i.e., Hirsh, 1948; Hirsh \& Webster, 1949; Webster, 1951; Jeffress et al, 1956). McFadden (1966), however, studied the effects of continuous vs pulsed noise maskers in homophasic and antiphasic conditions (see also Watson, 1962). McFadden used a $400-\mathrm{Hz}$ tone of $125 \mathrm{msec}$ duration as the signal and a wide-band thermal noise as the masker. In the pulsed condition, the noise and the signal onsets and offsets were simultaneous. He found that, in the homophasic condition, $\mathbf{N}_{\mathrm{o}} \mathrm{S}_{\mathrm{o}}$, signal detectability when the noise was pulsed was the same as it was when the noise was continuous. However, signal detectability in the antiphasic conditions ( $\mathrm{N}_{0} \mathrm{~S}_{\pi}$ and $\mathrm{N}_{\pi} \mathrm{S}_{\mathrm{o}}$ ) was degraded when the masker was pulsed relative to the conditions in which the masker was continuous, resulting in a smaller MLD (an MLD, or masking level difference, is the advantage exhibited by the binaural system when detecting a tonal signal in a background of masking noise in antiphasic relative to homophasic stimulus conditions).

In the second part of this study, McFadden investigated the effect of extending the duration of the masker forward in time (prior to the onset of the signal) from the pulsed condition just described. The masker and signal offsets occurred simultaneously, but the onset of the masker preceded the onset of the signal. The results indicated that the MLDs increased from about $12 \mathrm{~dB}$ at $0 \mathrm{msec}$ of forward "fringe" to about $16 \mathrm{~dB}$ at $600 \mathrm{msec}$ of "fringe," the value of the MLD that was obtained in continuous noise.

McFadden explained the results of this study in relation to the Webster-Jeffress hypothesis (Webster, 1951; Jeffress, Blodgett, Sandel, \& Wood, 1956; Jeffress, 1972). He suggested that the continuous noise condition provided the binaural auditory system with a baseline or reference phase prior to the onset of the signal. At the onset of the signal, then, the resultant phase shift $(\theta)$ was from a known interaural phase to an interaural phase relation that was dependent upon the characteristics of the signal. In the pulsed masker condition, however, there was no interaural phase relation prior to the onset of the signal since there was no "noise alone." The noise and the signal were added prior to presentation so that the phase shift caused by the simultaneous presentation of the two waveforms was not away from a reference phase and was therefore less discernible.
In a recent study investigating backward masking, Dolan and Trahiotis (1972) used a masking noise of fixed duration ( $75 \mathrm{msec}$ ) to mask an $8-\mathrm{msec}$ tone in order to determine the effect of various temporal relations between signal and masker on the $\operatorname{MLD}\left(\mathrm{N}_{0} S_{0}\right.$ relative to $\left.N_{0} S_{\pi}\right)$. They varied the temporal relations between the tonal signal and the noise masker by delaying the onset of the noise relative to the onset of the signal. One of their conditions, $\Delta t=0$, is comparable to the pulsed masker condition studied by McFadden except for the $67 \mathrm{msec}$ of masker occurring after the offset of the signal. It is interesting to note, however, that the size of the MLD obtained by Dolan and Trahiotis in this condition was $20 \mathrm{~dB}$, whereas McFadden's pulsed masker condition yielded an MLD of only $12 \mathrm{~dB}$. Even though it has been shown that, as the duration of a tonal signal decreases, the magnitude of the MLD increases (Blodgett, Jeffress, \& Taylor, 1958), an MLD of the magnitude obtained by Dolan and Trahiotis would not be expected. This is so because the maskers used in their conditions were gated and, unlike those used by McFadden, had no forward fringe. Evidently, then, the occurrence of part of the masking noise after the offset of the signal in the Dolan and Trahiotis study may have acted much like the forward fringe in the McFadden study.

These data may be interpreted as evidence that the binaural system uses the occurrence of the masking noise after the offset of the signal to provide a baseline or reference phase similar to that discussed by McFadden, 1966). Thus, the phase shift of the signal-plus-noise waveform might become more apparent and result in higher detectability and a larger MLD. The present study was an attempt to determine the extent to which occurrence of masking noise after the offset of the signal improves performance in a binaural detection task.

\section{PROCEDURE}

The binaural masking conditions investigated in this study were the homophasic condition, $N_{0} S_{o}$, and the antiphasic condition, $\mathbf{N}_{\mathbf{0}} \mathbf{S} \pi$. The signal was a 20-msec sample of a $500-\mathrm{Hz}$ sinusoid gated with $2.5-\mathrm{msec}$ rise-fall times. The masker was a band-pass noise, $100-3,000 \mathrm{~Hz}$, with a spectral level of $50 \mathrm{~dB}$ SPL. Masker durations were $30,50,250,500$, and $1,000 \mathrm{msec}$. All pulsed maskers were gated with 1-msec rise-decay time. In addition, a continuous masking condition was employed. Three of the six temporal relations between signal 


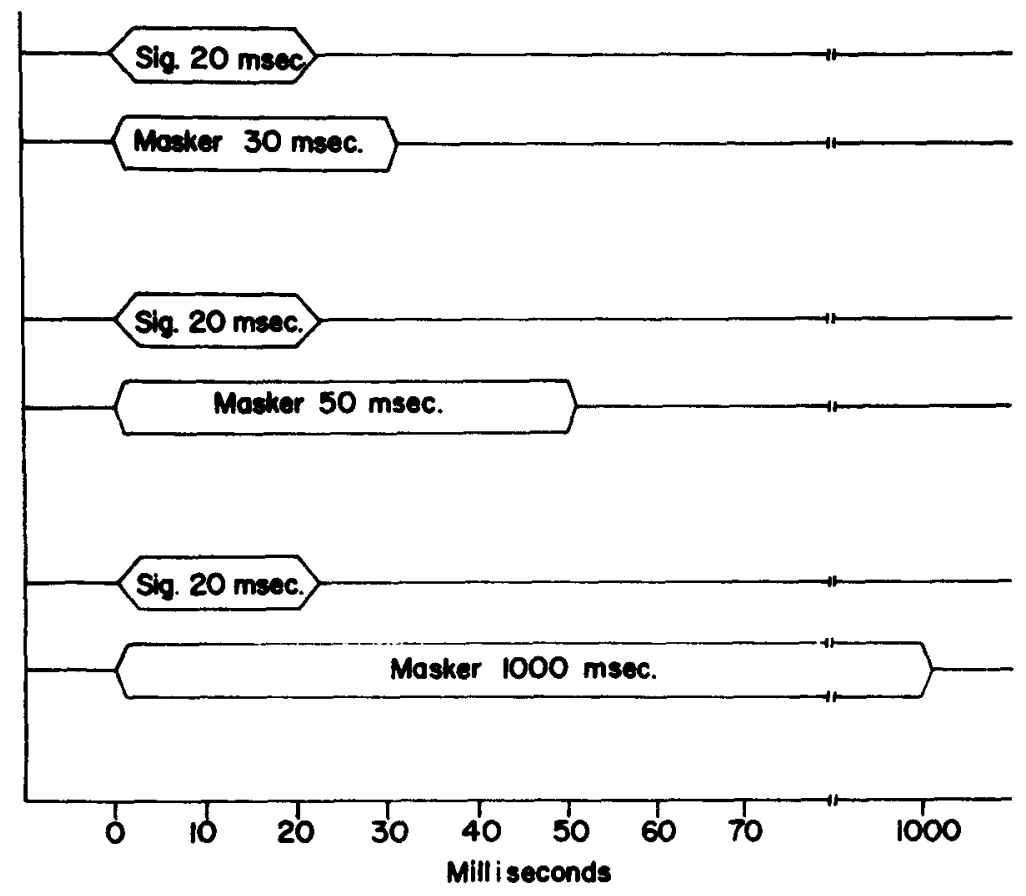

Fig. 1. Three of the six temporal relations of the signal and the noise investigated in the present experiment. In all conditions, the signal was a $20-\mathrm{msec} 500-\mathrm{Hz}$ tone. In addition to the masker durations of 30,50 , and $1,000 \mathrm{msec}$ shown here, $250 \mathrm{msec}, 500 \mathrm{msec}$, and continuous maskers were also employed. Rise-fall times were 2.5 msec for the signal and 1 msec for the noise masker.

and masker considered in this study are shown in Fig. 1.

To measure performance in each masking condition and to determine the magnitude of improvement in the antiphasic condition relative to the homophasic condition, a two-alternative temporal forced-choice (2ATFC) technique was used. The O's task was to state in which of two observation intervals the signal had occurred. Each trial consisted of the following temporal sequence: warning light $(0.4 \mathrm{sec})$, pause $(0.4 \mathrm{sec})$, light indicating first observation interval (0.02 sec), pause $(0.4 \mathrm{sec})$, light indicating second observation interval $(0.02 \mathrm{sec})$, response interval $(1.5 \mathrm{sec})$, and "feedback" interval ( $0.2 \mathrm{sec})$. The lights used to designate the intervals were spatially and temporally discrete. The signal occurred on every trial with equal probability of occurrence in each interval. The feedback light informed the $O$ after each trial which of the two intervals had contained the signal.

The measure of performance for each masking condition was the average percent correct, $\mathbf{P}(\mathbf{c})$, for Intervals 1 and 2 computed for 400 trials at each of two signal-to-noise ratios. From these data, 2-point psychometric functions (functions relating percent correct to signal energy) were generated for each $\mathrm{O}$ at
$d^{\prime}=m\left(E / N_{0}\right)^{k}$, where $E$ is the energy of the signal, $\mathrm{N}_{0}$ is the noise power per unit bandwidth, and $m$ and $k$ are constants. $d^{\prime}$ was transformed to $P(c)$, $\left(\mathrm{E} / \mathrm{N}_{\mathrm{o}}\right)$ was transformed to $10 \mathrm{log}$ $\left(\mathrm{E} / \mathrm{N}_{\mathrm{o}}\right)$, and the best-fitting values of $m$ and $k$ were selected by eye. A more detailed description of this procedure has been reported by Egan (1965).

The four Os used in this experiment were either members of the staff of the Parmly Hearing Institute or students at Loyola University having clinically normal hearing; they were paid for their services.

\section{RESULTS}

Twelve psychometric functions were determined for each $O$. These functions correspond to the 12 combinations of the two interaural phase relations and the six masker durations. As has been shown by Egan (1965), the slope of the psychometric function may be considered a constant for a single $O$ across all conditions. The values of $k$, the slope of the function, which best fit the data were 1.25 for one of the $O s$ and 1.00 for the other three.

The size of the MLD in decibels for each masker duration was computed by measuring the lateral displacement of an $N_{0} S_{\pi}$ function from its corresponding $\mathrm{N}_{\mathrm{o}} \mathrm{S}_{\mathrm{o}}$ function. Since the inter- $O$ variability was small, only the mean MLDs for the four Os are reported in Fig. 2 as a function of masker duration.

As indicated in Fig. 2, as the

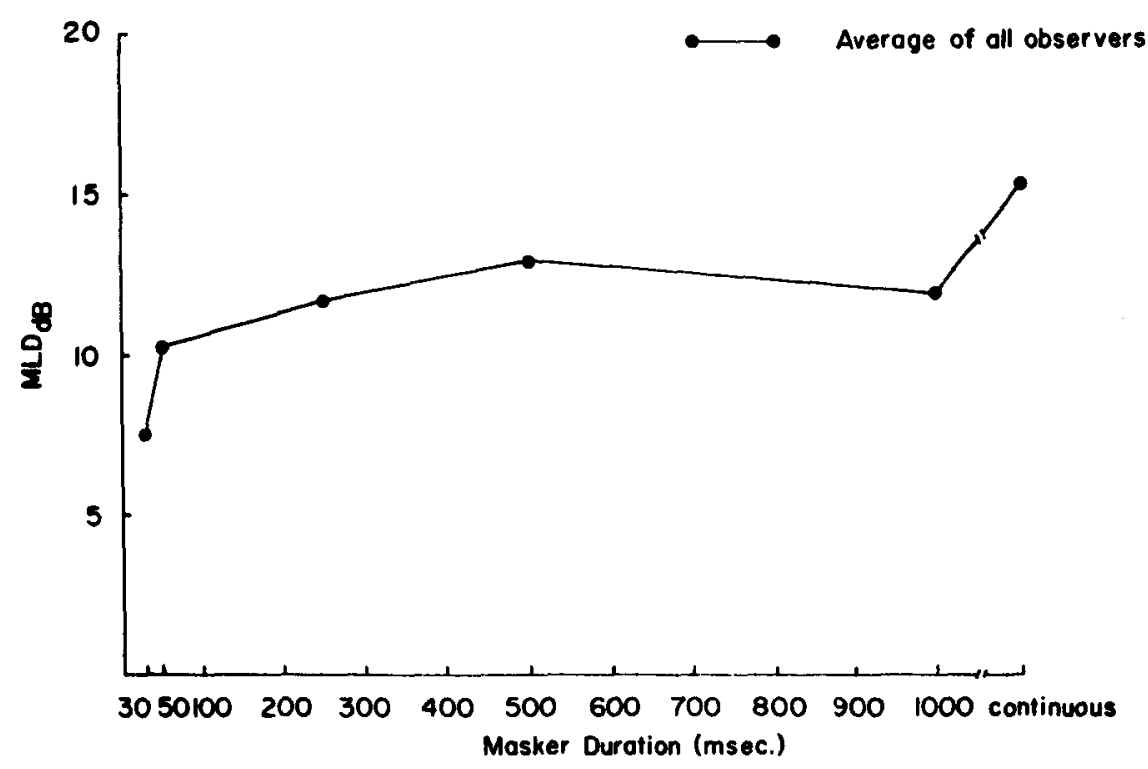

Fig. 2. The magnitude of the mean MLD $\left(N_{0} S_{\pi}\right.$ relative to $\left.N_{0} S_{0}\right)$ for the four Os as a function of masker duration. Each point represents the magnitude of the mean MLD based on the data for the four Os at one of the six temporal relations investigated. 
duration of the masking noise increases from $30 \mathrm{msec}$ to continuous, the mean MLD increases from 7.50 to $15.50 \mathrm{~dB}$. Of this total increase of $8 \mathrm{~dB}$, roughly half $(4.25 \mathrm{~dB})$ occurs between the 30- and 250-msec masker conditions. As the masker duration increases from 250 to $1,000 \mathrm{msec}$, no noteworthy change in the mean MLD takes place. However, in the change from the $1,000-m s e c$ duration to continuous masking noise, the mean MLD increases $3.50 \mathrm{~dB}$. Therefore, increase in the mean MLD takes place in the transition between masker durations of 30 and 250 msec and also in the shift from the $1,000-$ msec masker condition to the continuous masker condition. However, the increases in masker duration from 250 to $1,000 \mathrm{msec}$ had little effect on the mean MLD.

Since the size of the MLD is determined by measuring the lateral displacement of the homophasic and antiphasic psychometric functions, it is apparent that the size of the MLD can be altered by changes in the position of either the $N_{0} S_{0}$ function or the $\mathrm{N}_{0} \mathrm{~S}_{\pi}$ function, or both. In order to examine the basis for the change in the MLD obtained as masker duration was manipulated, the mean signal-to-noise ratio necessary for $80 \%$ correct detection for the four Os was calculated for each combination of interaural phase relations and masker duration. These data are presented in Fig. 3. Since a theoretical function of the same slope can be fitted rather well to all the psychometric functions for a given $O$, the MLD is independent of the level of performance. Therefore, the vertical displacement of the $N_{0} S_{o}$ function from the $N_{0} S \pi$ function is the MLD.

It should be noted that as masker duration increases from 30 to $250 \mathrm{msec}$, there is both a small improvement in performance under the antiphasic condition and a small decrement in performance under the homophasic condition. The net effect of changes in performance in both the homophasic and antiphasic conditions is a 4.75-dB increase in the magnitude of the MLD. The increase in the mean MLD between the 1,000-msec and the continuous masker condition is, however, almost solely the result of an improvement in performance under the $\mathbf{N}_{0} \mathbf{S} \pi$ condition.

\section{DISCUSSION}

The results of this study indicate that the magnitude of the MLD obtained when the signal and masker onsets are simultaneous depends upon the length of time by which the offset of the masker follows the offset of the signal.

Although the effect of this

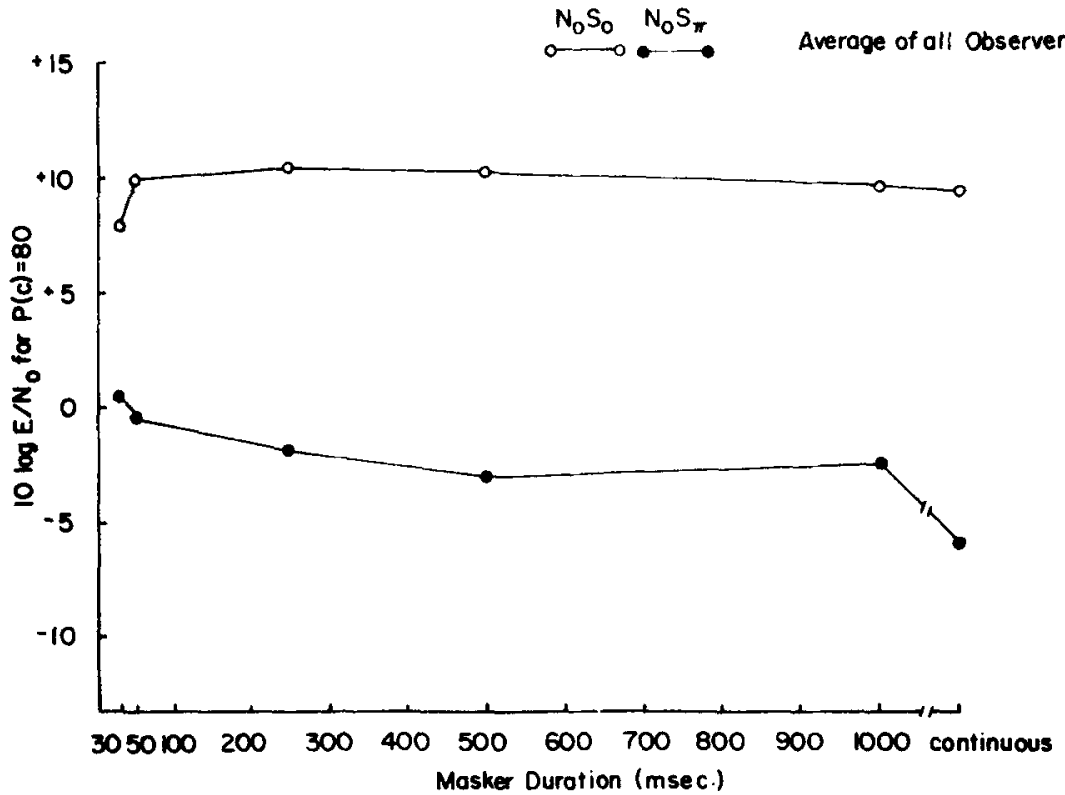

Fig. 3. Mean signal-to-noise ratio necessary for $80 \%$ correct detection $\left(\mathrm{N}_{0} \mathrm{~S}_{0}\right.$ and $N_{0} S \pi$ ) for the four $O s$ as a function of masker duration. Each point represents the mean signal-to-noise ratio for $80 \%$ correct detection based on the data for the four $O$ s at one of the six temporal relations investigated. Open symbols represent the $\mathrm{N}_{0} \mathrm{~S}_{0}$ data for a particular temporal relation. Closed symbols represent the $\mathrm{N}_{\mathrm{o}} \mathrm{S} \pi$ data for a particular temporal relation.

"backward fringe" on the MLD is somewhat similar to the effect of the forward fringe as reported by McFadden, there are some important distinctions that should be emphasized. In the present study (see Fig. 3), there was little change in the magnitude of the MLD between the 250- and the 1,000-msec masker conditions. However, as McFadden extended his masker forward in time, the obtained MLD increased steadily until, at 1,000 msec of forward fringe, it closely approximated the MLD obtained in the continuous masker condition. Therefore, McFadden was able to approximate the MLD obtained in the continuous masker condition by adding sufficient forward fringe to his pulsed masker. The results of the present study indicate that no amount of backward fringe leads to an MLD similar in magnitude to that obtained with a continuous masker.

In a recent study, Robinson and Trahiotis (1971) investigated the effect of forward fringe on MLDs by masking tonal signals of various durations with pulsed noise maskers. As in the McFadden study, the offsets of the signal and masker occurred simultaneously, and the onset of the masker was moved forward in time relative to that of the signal. Their results indicated that the effects of forward fringe on the magnitude of the MLD varied as a function of signal duration. At brief signal durations (i.e., $32 \mathrm{msec}$ ), the magnitude of the MLD changed considerably as the duration of forward fringe was varied. At longer signal durations (i.e. 256 msec), however, the duration of forward fringe had little effect on the magnitude of the MLD. In order to compare the magnitude of the forward fringe effect to that of the backward fringe effect, the data from the present experiment are compared to the data obtained by Robinson and Trahiotis when a brief tonal signal was employed.

In Fig. 4, the data from Fig. 3 are replotted as a function of noise fringe rather than masker duration. Thus, a masker duration of $30 \mathrm{msec}$ is represented by a noise fringe of $0 \mathrm{msec}$ (including the rise-fall times of the signal and noise), a masker duration of $50 \mathrm{msec}$ is represented by a noise fringe of $20 \mathrm{msec}$, and so on. Also plotted in Fig. 4 are the relevant data from the Robinson and Trahiotis study.

It can be seen from the upper set of points that performance in homophasic conditions when forward fringed maskers are employed is similar to performance when backward fringed maskers are employed. The only discrepancy between the two sets of data is a 2 -dB improvement in performance obtained in the 0 -msec fringe condition of the present study. This 2-dB difference represents a small improvement in performance relative 


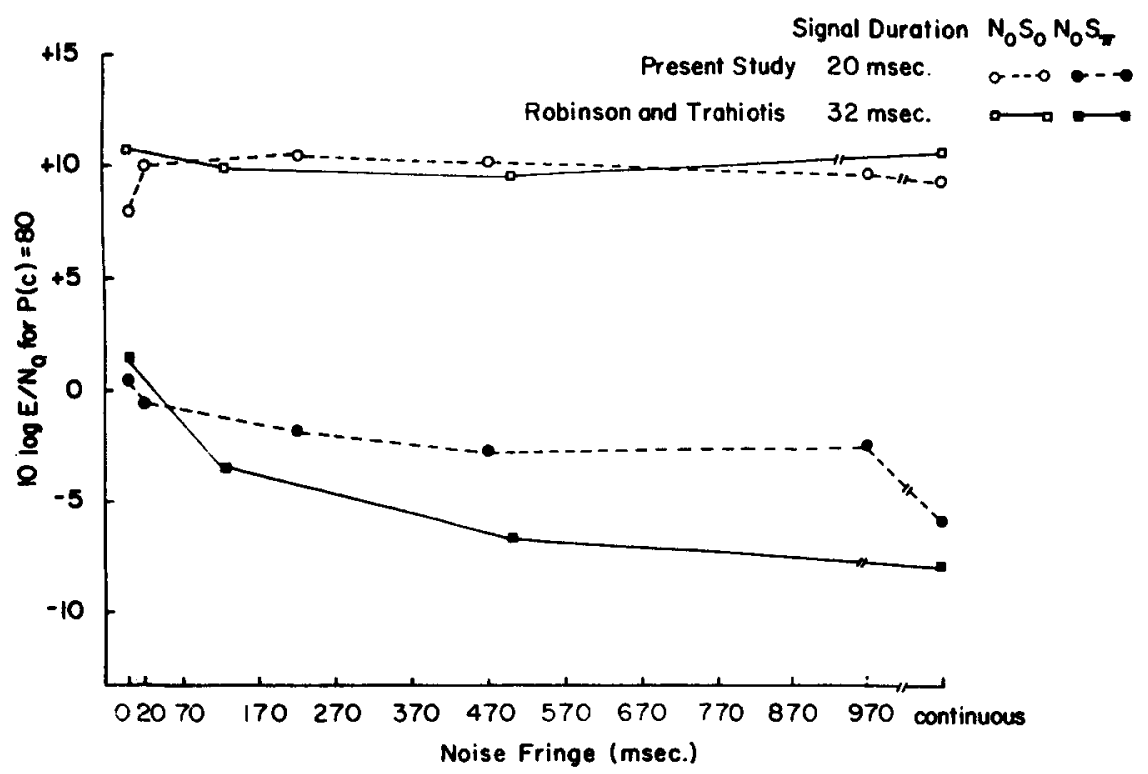

Fig. 4. Data presented in Fig. 3 replotted as a function of noise fringe along with similar data from the Robinson and Trahiotis study. The data from the present study (dotted line) are plotted as a function of backward noise fringe. Therefore, a noise fringe of 0 msec corresponds to a masker duration of $30 \mathrm{msec}$; a noise fringe of $20 \mathrm{msec}$ corresonds to a masker duration of $50 \mathrm{msec}$, and so on. The data from the Robinson and Trahiotis study (solid line) are plotted as a function of forward noise fringe. Open symbols represent the data from the $\mathrm{N}_{\mathrm{o}} \mathrm{S}_{\mathrm{o}}$ condition, while closed symbols represent the data from the $N_{0} S_{\pi}$ condition. The signal duration for the Robinson and Trahiotis data was 32 msec.

to other homophasic conditions when the signal and noise had simultaneous onsets and offsets. Neither McFadden nor Robinson and Trahiotis found any change in performance under homophasic conditions as a function of temporal relations of the signal and masker. Further investigation of the 30- and 50-msec masker duration conditions in our laboratory, using three other Os, yielded results consistent with those obtained in the present study. Although this difference appears to be reliable, its causes are not clear.

Examination of the lower set of points in Fig. 4 indicates that the performance in the antiphasic conditions seems to differ as a function of whether the fringe is in the forward or in the backward fringe direction. Additionally, performance in the forward fringe conditions approaches that obtained in a continuous masking situation as the duration of fringe increases. When the forward fringe is $500 \mathrm{msec}$, for example, the magnitude of the MLD is essentially the same as that obtained in the continuous masking situation. These results are not obtained in the backward fringe conditions. Performance in the antiphasic masking conditions improves only $3 \mathrm{~dB}$ as the backward fringe increases from 0 to $970 \mathrm{msec}$, and the magnitude of the MLD in the 970-msec backward fringe condition is considerably less than that of the MLD obtained when a continuous masker was used. This difference is indicative of the inability of backward fringe to approximate the effect of a continuous masker on the magnitude of the MLD.
In summary, our data indicate that detectability in forward and backward masker fringe conditions is not equivalent, although both may cause an enhancement in performance. A masker onset $1,000 \mathrm{msec}$ prior to the onset of the signal yields performance comparable to performance obtained when a continuous masker is used. It appears that no amount of backward fringe will produce results equivalent to those found in continuous noise.

\section{REFERENCES}

BLODGETT, H. C.. JEF FRESS, L. A., \& TAYLOR, $R$. W. Relation of masked threshold to signal-duration for various interaural phase-combinations. American Journal of Psychology, 1958, 71, 283-290.

DOLAN, T. R., \& TRAHIOTIS, C. Binaural interaction in backward masking. Perception \& Psychophysics, 1972, 11, 92-94.

EGAN, J. P. Masking-level differences as a function of interaural disparities in intensity of signal and of noise. Journal of the Acoustical Society of America, $1965,38,1043-1049$.

HIRSH, I. J. Influence of interaural phase on interaural summation and inhibition. Journal of the Acoustical Society of America, 1948, 20, 536-544.

HIRSH, I. J., \& WE BSTER, F. A. Some determinants of interaural phase effects. Journal of the Acoustical Society of America, 1949, 21, 496-501.

JEFFRESS, L. A. Binaural signal detection: Vector theory. In J. V. Tobias (Ed.), Foundations of modern auditory theory. New York: Academic Press, 1972.

JEFFRESS, L. A.. BLODGETT, H. C.. SANDEL, T. T., \& WOOD, C. L., III. Masking of tonal signals. Journal of the Acoustical Society of A merica, 1956, 28, 416-426.

MCFADDEN, D. Masking-level differences with continuous and with burst maskin noise. Journal of the Acoustical Society of America, 1966, 40, 1414-1419.

ROBINSON, D. E., \& TRAHIOTIS, C. Effects of signal duration and maskex duration on detectability under diotic and dichotic listening conditions. Journal of the Acoustical Society of America. $1971,49,102$.

WATSON, C. S. Signal detection and certain physical characteristics of the stimulus during the observation interval. PhD thesis, Hearing \& Communication Laboratory, Indiana University.

WEBSTER, F. A. Influence of interaural phase on masked thresholds. I. The role of interaural time-deviation. Journal of the Acoustical Society of America, 1951, 23, $452-462$.

(Received for publication June 12, 1972.) 